\title{
Le souffle de l'histoire chez Georgette LeBlanc
}

Julie ST-LAURENT, Université de Toronto

Le travail de Georgette LeBlanc suit le chemin ouvert par l'écriture poétique des femmes en Acadie, qui, depuis les années 1980, selon François Paré, a donné à lire « une subjectivité qui ne pouvait plus être réductible à ses seules références communautaires»(Distance, 201). LeBlanc en partage le souci de la forme textuelle, la mise en question de l'unité identitaire et un certain intimisme au féminin. Mais cette filiation n'est pas tout à fait déterminante, tant son œuvre s'élabore avec le plus grand souci de l'histoire, à laquelle la génération précédente de femmes poètes a voulu tourner le dos, au moins en partie, «esquiv[ant] la question du destin national » (Paré, Distance, 205). Chez l'auteure dont il est question ici, pas de telle mise entre parenthèses. Il s'agit de raconter des histoires à partir d'une histoire commune, d'où sa volonté de présenter ses livres, Alma (2006), Amédé (2010), Prudent (2013) et Le grand feu 2016), en tant que « romans poétiques » (LeBlanc, «Department of French »), dont la valeur narrative devient essentielle. LeBlanc revisite différents moments de l'histoire acadienne au fil de ses écrits : le passage à la modernité de l'Acadie (Alma), les premiers enregistrements de la musique cadienne en Louisiane (Amédé), la révolte des déportés du navire Pembroke (Prudent) et l'incendie de Baie Sainte-Marie en 1820 (Le grand feu).

La langue acadienne de la Baie Sainte-Marie (Nouvelle-Écosse) est la matière culturellement signifiante à partir de laquelle LeBlanc développe son écriture poétique singulière, qu'elle ne veut pas «prescriptive » (Grand feu, 89) : c'est sa langue affective, sa plus belle langue, affirme-t-elle en entrevue dans l'Acadie Nouvelle (« Georgette LeBlanc lance son $4^{\mathrm{e}}$ roman poétique »). En effet, cette langue poétique possède une sensibilité très vive, autant pour dire l'émotion que la sensation, exprimant depuis le tout début « le braquement ${ }^{1}$ du désir » (Alma 11$)$ à travers la vie de différents personnages qui ont existé dans le temps. L'écriture corporelle que la poète développe permet de mettre en dialogue les sujets représentés et l'histoire, qui n'a plus rien d'abstrait - et aussi de l'excéder, puisque l'expérience subjective ne peut complètement être assimilée à la mémoire générale.

Je montrerai ainsi comment s'entrelacent dans sa poésie narrative le temps vécu - celui du sujet, qui ressent au présent - et le temps du monde - celui de 1 'histoire $-^{2}$, grâce à un 
ensemble de stratégies de présentification de l'écriture, qui font appel à l'expérience du corps et qui soulignent le processus créateur en cours. Cet article se révèle beaucoup trop court pour rendre justice à la richesse des quatre livres de LeBlanc ici étudiés, mais j'aimerais au moins qu'il puisse montrer l'unité profonde de son œuvre, où l'énergie de la vie triomphe sur la souffrance reliée au passé douloureux du peuple acadien marqué par la Déportation. En effet, les quatre livres publiés révèlent une voix unique, modulée par des vers libres et sensuels, qui reformule l'histoire, en écrit une autre (Grand feu, 89), suivant cette volonté plusieurs fois réitérée par LeBlanc ${ }^{3}$, et qui montre la nature fondamentalement inachevée et inachevable de la mémoire. « [L]e présent est un énorme cadeau rempli d'expériences » («Georgette LeBlanc... et Georgette Leblanc», 16), affirme la poète en jouant de la polysémie du mot «présent», signifiant à la fois «maintenant» et «don», ce qui laisse poindre toute la richesse de son écriture pénétrée de l'énergie de l'instant. Dans cette idée, nous verrons comment cette poésie qui se fait historienne «libèr[e] les potentialités du présent », tel que Paul Ricœur («Fonction narrative », 66) l'a affirmé au sujet de la fonction narrative dans le récit fictif et l'écriture historique.

La réflexion menée dans ces quelques pages profite largement de la récente publication du Grand feu, livre qui explicite les motivations du travail d'écriture de LeBlanc en mettant en scène de façon magistrale la venue à l'écriture de Cécile Murat, jeune fille qui a vécu au dixneuvième siècle et qui s'est fait connaître à travers l'œuvre fictive de l'écrivain et historien Alphonse Deveau, le Journal de Cécile Murat : LeBlanc la transforme en poète. L'auteure avoue à propos de son livre que «[c]'est l'histoire de ce que ç'a pris pour écrire Alma» («Georgette LeBlanc lance son $4^{\mathrm{e}}$ roman poétique »), texte qui a proposé une nouvelle figure mythique féminine heureuse et libre dans son corps ${ }^{4}$. D'Alma à Cécile, l'expérience du féminin se voit inextricablement liée à l'expérience créatrice, ce qui invite à porter attention à l'empreinte du corps sexué dans cette poésie qui conjugue écriture du temps et écriture de la sensualité.

\section{Des corps vécus}

Les trois premiers livres de LeBlanc, ayant pour titres les prénoms des personnages qui seront présentés dans l'écriture (Alma, Amédé et Prudent), ont d'emblée mis en relief l'aspect vécu de l'histoire qui sera remise en mémoire. Ce centrage du regard sur l'individu indique le déplacement de perspective qu'opère l'écriture de la poète. L'acte de mémoire est ramené à sa 
source même, le sujet, ce que la forme essentiellement pronominale du verbe «se souvenir » met en relief, comme le rappelle Ricœur (Mémoire, 115) : l'écrivaine, de même que les lecteurs et lectrices, se souviennent donc avec les personnages, mélangeant présent révolu de l'histoire au présent de l'énonciation.

Les sujets et leur relation particulière au monde sont placés au premier plan de l'écriture et cette relation possède une composante immanquablement incarnée, chez LeBlanc : le corps y constitue à la fois un lieu de mémoire, où elle prend forme et reprend vie, et un «instrument de jouissance et de découverte » (LeBlanc, «La chair des mots »). Suivant cette idée, écrire s'y révèle un geste fondamentalement corporel, en phase avec le désir. L'écriture naît de sensations particulières ressenties dans le secret de la chair, ce que présentent les premières pages du Grand $f e u$, alors que Cécile revient de chez son amoureux Jean-Baptiste : «Cécile sentait les braises de la nuit / jusqu'à dans son corps, son temple à elle » (19). Cette chaleur fait écho à toutes les sensations qui ont tissé les livres précédents, témoignant de «la sensualité complexe du "je" sujet » («Georgette LeBlanc...», 16), diffractée par les expériences de vie mises en écriture. Aussi les métaphores et comparaisons qui font appel au corps vécu et sentant, abondent-elles dès le début: «[1]es bras [de la petite Alma] et ses mains et ses pieds / arrêtiont point de nager / comme une picogie ${ }^{5}$ »(12); dans Amédé, «son corps de chêne fier» (56) évoque une force vitale; dans Prudent, l'Aîné capte l'attention grâce à «sa voix épaisse / comme les racines » (18). Les sensations exploitées témoignent souvent d'une appartenance à la nature, au territoire, comme l'explicitent «les racines », tout en permettant d'affleurer l'affectivité des personnages, leur singularité. Cet éloge du sensible culmine dans une sorte d'érotisme existentiel qui traverse l'écriture de la poète, résumée par l'intrigante métaphore des «skirts du ciel » (Amédé, 47), qui apparaît tel un leitmotiv dans son deuxième livre. Aussi les personnages mis en écriture sont-ils tous des êtres désirants, à la sensualité heureuse, au moins pendant un moment : Alma devient « un autre mode de bêtes » avec Pierrot (62) ; « dans chaque pétale de Rose / dans chaque douce épaisseur de sa voix / Amédé retrouvait mémoire, racines » (38); Prudent «avai[t] trouvé [s]a Femme. / Brune comme la terre. / Elle l'est encore » (30); en pensant à son Jean-Baptiste nageant nu, Cécile sentait « la flamme qui montait» (54). Le désir ressenti donne des « racines » qui se nourrissent du présent, comme la «flamme» que Cécile éprouve devient la flamme créatrice : «le désir, l'amour, le territoire et l'écriture se rejoignent ainsi en un continuum fragile » (Papillon), que la poésie de LeBlanc s'efforce de magnifier. 
C'est souvent dans l'écriture de la sensation, très sensible au territoire, que la langue de LeBlanc dévoile toute sa profondeur poétique, comme lorsqu'Alma réfléchit à sa relation avec Pierrot : « ej grimpe mes fleurs sauvages / il continue à suivre » (29). Ainsi, malgré que LeBlanc souligne elle-même la nature fondamentalement narrative de ses écrits, le discours poétique qui les traverse, leur confère une certaine opacité, qui témoigne d'une prise de distance avec le référent premier. Ils racontent quelque chose qui s'évapore par moments dans le travail de la langue: depuis Alma, «l'expression des événements, des pensées et des émotions des personnages recourt à des figures de style et conserve une part d'ambiguïté et de polysémie » (Lacombe 62). Aussi faut-il remarquer qu'Alma s'ouvre sur «un éloge de la brume» caractéristique de Baie Sainte-Marie (Saada 52), qui embrasse la vie : « elle [la brume] avance et grouille tranquille / comme si elle était à sa place partout » (10). La personnification de la brume témoigne du besoin de mouvement, libre, sans besoin de se justifier, celui du langage poétique de LeBlanc refusant d'être statique, univoque, au profit d'une authenticité d'expression qui met son voile sur le récit loin d'être seulement historique. Ce mouvement rappelle que le corps vécu, «à sa place partout» dans l'écriture, travaille de l'intérieur la forme narrative: l'expérience corporelle constitue un surplus de vie que la pratique poétique cherche à rendre dans le langage, brouillant toutes catégories.

Bien qu'un plaisir de l'écriture soit manifeste chez LeBlanc, il pointe souvent des moyens d'expression qui excèdent la mise en mots, ce que la récurrence du cri évoque dans Alma et Amédé, de même que la présence de la musique à travers son œuvre. Alma fait référence à la musique noire américaine (le blues de Big Mama Thornton et le gospel); Amédé raconte l'histoire de deux musiciens louisianais, Amédé Ardoin, chanteur et accordéoniste, et Angelas Lejeune, accordéoniste qui l'accompagne (Nyela 264); dans Prudent et Le grand feu, le rapport à la musique est plus subtil, la voix de Prudent résonnant comme un «tambour sacré » (63) et le parcours de Cécile «feel comme dla bass » (73). La musique mais aussi la danse sont des figures d'harmonie au sein de l'écriture de la poète, qui témoignent d'une proximité à soi, à son corps et son âme inséparables. De façon touchante, l'écriture hybride de LeBlanc - où la chair dialogue avec l'histoire - outrepasse les dualités habituelles, comme en témoigne ce passage marquant d'Alma, où l'écriture est associée à la danse :

j'ai braqué à écrire des histoires

ej sais point trop ça qu'elles voulont dire encore

mais j'aime de voir mes mains grouiller ${ }^{6}$ sur la page 
comme si mon corps et ma tête et mes mains

étiont tous manière de la même personne (47)

Il se crée dans l'œuvre de LeBlanc un réseau écriture-danse-musique, ces trois pratiques artistiques répondant l'une de l'autre, donnant une matière incarnée à l'écriture qui recompose le corps aux sens qui se mélangent : «[1]a lumière dans les pieds. Les yeux dans le corps » (Alma, 43). Cet entrelacement permet aussi d'élargir l'espace d'expression, renvoyant à des univers où la trace littéraire n'existait pas, puisqu'il faut parler au corps, «chanter pour faire danser / sans mots »(Amédé, 22) ${ }^{7}$, inviter à bouger. Amédé approfondit le lien vital qui s'établit entre l'expérience humaine et l'expérience artistique par l'entremise de la musique: lors d'une tempête, « les jambes d'Amédé jusqu'à ses genoux s'aviont mouillé / et les bras encerclés autour de sa Cordine, à lui / pour garder ses poumons secs » (42). Le livre exploite la polysémie des «poumons », qui désignent ici à la fois le corps d'Amédé résistant à l'inondation et aussi le soufflet de l'accordéon: ce dernier permet de respirer, parle au corps dans ses fonctions premières. D'une façon analogue, dans Le grand feu, la poète explicite le lien entre la musique et plus largement l'art, en tant que langage incarné - et l'expérience humaine : la réussite de Cécile se termine dans une musique profonde, «[ç] feel comme dla bass » (titre de la dernière section du livre). Dans un glossaire créatif où elle explique ses «matières » poétiques, LeBlanc, à l'entrée « bass », joue de l'homonymie de ce mot anglais, donnant la définition suivante : «La fondation individuelle et spirituelle qui est aussi nécessairement matérielle puisque j'avons encore le privilège d'habiter des corps humains et imparfaits » (83). Ainsi, la basse musicale devient un fondement, un support fondamental («base »), qui témoigne d'une condition humaine «nécessairement matérielle », profondément incarnée, nourrie de mouvement et de sensations. À de multiples reprises dans son écriture à sensibilité féministe, qui «challenge [the] mind/body dualism » (Ahmed et Stacey 4), LeBlanc invite à goûter « la peau juteuse du sacré » (Prudent, 118), l'expérience humaine et sa quête de sens prenant ancrage dans l'expérience sensuelle du monde.

Afin de repenser l'histoire acadienne, la poète donne une chair vivante à ses personnages, qui leur permet de sentir, d'aller à la rencontre du monde, de tisser avec lui un nouveau rapport. Les injonctions formulées par Prudent témoignent de l'intrication singulière d'une quête de liberté et d'une quête de sensualité dans l'écriture de LeBlanc : «Il faut prendre le bote ! / Il faut reprendre nos corps!»(105; en italiques dans le texte). Le travail de l'auteure représente en 
effet une lutte contre l'aliénation corporelle, bien évidemment culturelle mais aussi à nouveau féministe. Si la poésie de LeBlanc représente différents corps vécus, elle s'inscrit d'abord dans l'expérience d'un corps singulier, situé et sexué, ce que l'écriture d'Alma a mis en relief. LeBlanc désire offrir une autre vision des femmes, qui n'ont jamais eu de véritable corps dans la littérature acadienne, et y réintégrer plus généralement de l'érotisme et de la sensualité ( La chair des mots »), qualités que l'on retrouve autant dans ses livres qui ont pour personnages principaux des hommes que des femmes. De façon révélatrice, Amédé débute par un prologue intitulé «Alma raconte » : ce niveau extradiégétique du poème narratif invite à garder à l'esprit que la mémoire offerte est travaillée de l'intérieur par « une éthique au féminin qui passe par le corps sexué » (Sing 168), telle que Pamela V. Sing l'a observée chez les écrivaines francoalbertaines Marguerite Primeau, Marie Moser et Jacqueline Dumas, c'est-à-dire un souci de donner une voix heureuse au désir qui s'articule, comme nous le verrons, à une réécriture de l'histoire «dialogique, polyphonique, déhiérarchisée, consciente du caractère partiel des généralisations » (168), qui cherche à rendre l'expérience vécue du temps, à montrer comment le passé parle au présent.

\section{L'écriture au présent}

L'écriture incarnée de LeBlanc empêche toute remémoration figée du passé, qui devient une variante du présent du corps éprouvant le monde, corps qui est celui de l'auteure, mais aussi celui du lecteur ou de la lectrice. Ce dialogue mémoriel est d'ailleurs accentué par l'alternance des temps de narration dans toutes ses œuvres, d'autant plus que certaines, Alma et Prudent, enchâssent narration au présent, à la première personne du singulier, et narration au passé, avec la distance de la troisième personne du singulier. Ce balancement assure le dynamisme de la voix poétique qui désire repenser l'histoire, tout en marquant la profondeur d'une mémoire vivace, comme en témoignent ces mots du sage Prudent : «Ej comprenais. Ej comprends encore » (41). La poéticité de l'écriture de LeBlanc, en plus de ce qu'elle exploite de l'expérience corporelle, joue un rôle similaire, en ce qu'elle ramène toujours le discours narratif occupé à un objet du passé au présent de l'énonciation, où le langage lui-même, son style et ses images se donnent tout autant comme objet de l'attention. Ainsi cette écriture comporte-t-elle une nature paradoxale qui nourrit sa puissance de transformation, comme l'observe Catherine Leclerc au sujet d'Alma: «Tout en collant de près au quotidien qu'elle décrit, au passé prélittéraire dont elle fait la 
chronique, elle [la langue] assume entièrement sa littérarité »(59), la venue à l'écriture n'étant pas seulement racontée mais vécue pleinement depuis le premier livre. De surcroît, la versification libre de cette écriture narrative qui cumule les contre-rejets, reprises et anaphores dévoile la profonde oralité de cette prise de parole littéraire, qui demeure langue vécue :
À Kespukwitk
la fortune se trouvait
si tu savais djetter
la braise qui elle, était
jusqu'au vent, jusqu'au souffle
jusqu'à l'énergie que ça prenait
pour la faire monter, parler
pour la refaire bruler (Grand feu, 16 ; 1'auteure souligne)

Dans cet extrait, l'usage de l'italique pour le «si » invite à prononcer avec une accentuation tonique le mot, tout comme d'autres manipulations typographiques au fil du livre, tel l'usage de la majuscule et des blancs, régissent la lecture qui appelle à la voix haute. De même, il faut remarquer qu'il est question du « souffle » nécessaire pour attiser le feu, celui qui naît des braises chaudes que Cécile a senties en elle : ce souffle se révèle physique et littéraire, ancré dans le tout présent de la vie.

Chez LeBlanc, retrouver ce présent où se constitue l'histoire - et se transmet, en l'occurrence, par l'écriture - permet d'éviter l'écueil d'une mémoire acadienne centrée sur la Déportation, dans laquelle la «souffrance [est] dev[enue] pierre angulaire d'une construction identitaire » («Department of French »). La poète ne veut collaborer à ce récit mémoriel qui rappelle trop de douleur en plus d'homogénéiser le passé, si bien qu'elle démonte «la clôture du récit [historique] qui [a été] mise ainsi au service de la clôture identitaire de la communauté » (Ricœur, Mémoire, 104). LeBlanc cherche à mettre en lumière la pluralité des expériences vécues au cœur de l'histoire acadienne, comme en témoignent ces questions soulevées en 2011 par rapport à l'idée de communauté, en l'occurrence la sienne :

Qui sont les auteurs de l'Histoire de cette communauté imaginée ? Quels événements considèrent-ils comme importants? Quels personnages deviennent héros et selon quels critères ? Où sont les héroïnes ? Comment faire pour ne pas perdre de Mémoire ces expériences culturelles qui ne correspondent pas aux schémas privilégiés ? («Georgette LeBlanc... », 15)

Les livres de LeBlanc, au fil de références et de mises en scène de personnages, font une part généreuse aux voix minorisées par l'histoire, qui n'ont pas eu la chance d'être véritablement 
entendues et représentées adéquatement. LeBlanc crée ses « héroïnes » acadiennes qui prennent la parole, qui écrivent, autrement absentes - «Évangéline, elle a pas dit grand-chose » (« La chair des mots »). Mais cette mémoire qu'elle refonde, touche à une communauté beaucoup plus vaste et qui se révèle faire partie de l'expérience acadienne : entre autres, c'est la servante Noemie, de Whylah Falls, communauté noire mythique de la Nouvelle-Écosse (imaginée dans le poème narratif du même nom de George Elliot Clarke [«Alma», 18]), qui encourage la révolte d'Alma ; c'est Amédé Ardoin, musicien créole, qui est dans les premiers à enregistrer la musique cadienne; ce sont les Mi'kmaqs avec qui les Acadiens vivaient en collaboration dans le passé raconté par Prudent et LeBlanc leur porte respect par sa façon d'utiliser les noms autochtones des lieux que son écriture fréquente, décolonisant ces espaces.

L'«autre » histoire que LeBlanc entreprend d'écrire, s'articule sur une somme de transformations qui se jouent sur le plan de l'identité, mais aussi à un niveau plus spécifiquement littéraire, celui du raconter. La poète est attachée au récit parce que l'intrigue qui le trame offre la possibilité d'une «transformation» («Department of French»), évoquant là une définition minimale et traditionnelle de la forme narrative - LeBlanc est attachée à l'idée du « roman »-, à laquelle elle donne une extension poétique au fil de son écriture. De fait, les quatre livres, organisés autour de différentes péripéties, se terminent sur l'arrivée à un temps nouveau, où l'horizon se révèle ouvert, empreint d'un espoir dont rend compte un lyrisme affirmé. « Alma avait retrouvé l'épaisseur de la terre. Elle avait du travail à faire » (112) : la métaphore terrestre témoigne du lien rétabli entre Alma et son territoire, d'une solidité garante de son épanouissement dans le futur, dans le «travail à faire ». On lit dans les derniers vers d'Amédé ce même avenir dégagé, parce qu' « il n'y avait rien de perdu // le Village s'avait refait » (81). Ainsi, la vie continue au terme de l'écriture qui transforme les traces de la mémoire : il « [...] restait rinqu'à guetter / que l'autre temps se faise » (44), au fil de l'écriture qui détruit et métamorphose les matières choisies par la poète. La narration dans l'écriture de LeBlanc progresse non pas vers une fin comme on pourrait s'y attendre, mais plutôt vers un commencement, vers un présent qui relance le récit, empêche sa véritable clôture.

Ainsi, faisant suite au récit de la révolte des déportés du navire Pembroke ${ }^{8}$, la section finale de Prudent, qui est un discours que l'Aîné adresse à son petit-fils, sort du cadre narratif au passé, mettant en valeur le présent de l'énonciation et toutes les potentialités et la sensorialité retrouvées dans l'instant vécu ${ }^{9}$ : 
Y a point de Paix.

Y aura plus de Paix.

Y a que la Vie.

Il faut monter et prendre, sentir,

mordre la chair comme la première fois,

gouter la sueur, le travail, le labeur que c'est de vivre. (117)

L'enchaînement des verbes d'action, qui engagent différents sens, témoigne d'un désir de retrouver une expérience authentique du monde, dans un rapport de proximité à ce dernier, mais aussi à soi - il faut «goûter la sueur » de sa propre présence. C'est cet appel au corps qui permet de poser le retour à une forme d'état originel de la vie. On peut aussi comprendre en ce sens l'importance de la nature au sein de l'écriture de LeBlanc, y inscrivant une autre forme d'énergie, non plus seulement humaine et jamais domestiquée ${ }^{10}$. Il faut renouer avec cette pulsion de vie initiale, « comme la première fois », disait Prudent : la conjonction «comme » est importante, car elle met en évidence l'entrée dans un nouvel ordre de référence, qui crée une «autre » vérité, se superposant à l'histoire connue. Ricœur situe dans cette suspension de la référence au monde commun autant la force (re)créatrice de la poésie que celle du récit, toutes deux exploitées chez LeBlanc : «Comme toute œuvre poétique la fiction narrative procède d'une épochè [mise entre parenthèses] du monde ordinaire de l'action humaine et des descriptions de ce monde ordinaire dans le discours ordinaire. La description doit être suspendue afin que la redescription prenne place. » («Fonction narrative », 57) Ce nouvel ordre de référence créé par l'usage singulier du récit fictif et du langage poétique chez LeBlanc fonde une mémoire alternative de l'expérience acadienne, en élargit les possibles, en cherchant à y intégrer la vérité du vécu subjectif, celui au cœur de son travail de la langue : «Tout ce qu'est vrai est ça que j'sens. Ça que j'feel » («Parée »), écrit la poète.

Livre qui réfléchit sur le geste de création, Le grand feu approfondit l'importance du temps et ainsi du présent dans la démarche de LeBlanc. En effet, le livre se construit autour de la mémoire de l'incendie de 1820 à la Baie Sainte-Marie et de Cécile Murat, devenue poète dans l'imaginaire de LeBlanc, moment historique qui permet à l'auteure de réfléchir sur la portée de l'écriture elle-même, comme elle l'explique : «J'ai comme brassé les cartes et j'ai raconté une autre histoire. Il y a un feu à la fin, mais c'est celui de la création » («Georgette LeBlanc lance son $4^{\mathrm{e}}$ roman poétique »). Le temps de l'écriture - temps vécu par excellence - devient une façon de remplacer le temps de la référence commune, scandé par l'histoire. Le feu qui a progressé au fil des pages, joue son rôle métaphorique de destruction et de purification, comme en témoignent 
les derniers vers qui pointent eux aussi sur un commencement : «le Grand Feu craquait / la lumière du feu dessinait têtes et corps nus / brulait pages, présages / il était une fois » (81). La synecdoque des «têtes et corps nus » évoque une chair sensible retrouvée, celle qui permet de sentir le vrai, et la sensualité qui parcourt l'œuvre de LeBlanc, tout en laissant poindre l'utopie d'individus réduits à un état minimal, libérés de l'emprise de l'histoire : le feu brûle «pages », le passé, et «présages », l'avenir tracé. L'écriture permet ainsi d'en finir avec l'histoire répétée : cette histoire devient littéralement à écrire, ouverture soulignée par la finale grandiose, « il était une fois », locution qui apparaît à plusieurs reprises au fil du Grand feu et qui constitue un archétype narratif qui invite à entrer dans un monde nouveau, à repartir à zéro. Le récit qui reconfigure la mémoire n'aura donc pas de terme.

L'immense brasier alimenté par l'écriture donne à sentir « la chaleur de la tempête, du Tyme / des présents débridés » (Grand feu, 81) : il y a à nouveau jeu d'homonymie, d'une part, d'une façon qui rappelle les propos cités en introduction, avec la polysémie des «présents », cadeaux de la vie, mais aussi temps multiplié, parce que le présent est au pluriel ; d'autre part, avec l'idée du «Tyme », qui signifie « grande fête » en langue acadienne, et qui résonne avec la préoccupation de la poète pour le temps (time). Justement, Le grand feu consacre quelques pages à la description d'une grande « Time Clock » (27-30) qui surplombe le lac où Cécile plongera à la fin du récit pour dire oui à l'écriture, plongeant pour ainsi dire au cœur du temps qui inexorablement s'écoule, symbolisé par «la Time Clock / la loi et le mystère, côté à côté » (30). Aussi peut-on penser que les multiples fêtes et réunions musicales qui ponctuent Alma et Amédé constituaient déjà une façon d'évoquer dans la trame narrative cette même jouissance du présent, suggérée par l'homophonie Tyme-Time. Le temps se révèle l'occasion de célébrer la vie et de s'épanouir, sans retenue, ce qui rappelle le plaisir d'exister des personnages de LeBlanc, fidèles à leur rythme intérieur : «mon corps grouille comme la musique / c'est le seul temps que ça fait de la suite » $(A \operatorname{lma}, 81)$. L'écriture de la poète, tout près du corps en tout instant, permet de reconfigurer le temps afin qu'il puisse parler maintenant, malgré l'histoire, malgré la mémoire, afin d'en refonder une réalité à toucher, et touchante, qui ne peut s'astreindre à être pure fiction : «elle voulait l'écrire / elle voulait le vivre » (Grand feu, 20 ; l'auteure souligne). La poésie au long souffle de LeBlanc, forme hybride nourrie d'un rythme dansant et continu, permet d'approcher l'énergie puissante au cœur de l'existence, comme l'évoque le poète québécois Marcel Labine: «Le long poème est l'une des formes du débordement: celui du monde sur 
notre propre personne, celui de l'Histoire qu'on ne peut raconter dans sa totalité, celui de la langue qu'on ne peut épuiser et, finalement, celui de la page qui ne peut le contenir » (127). La poésie ici étudiée affleure cet excès de vie, horizon de sens de l'écriture.

Le travail de LeBlanc évolue au fil d'une quête subjective, d'une quête artistique qui prend comme matière sa langue d'origine : l'auteure ne vise pas la reproduction d'une parlure et d'un univers particulier, mais recherche plutôt sa propre langue (« La chair des mots »), langue de poète qui exprimerait sa façon la plus intime d'être au monde, d'être au temps. Ainsi le temps de l'histoire reste-t-il indissociable du temps vécu, exploré à travers l'expérience du corps, qui confère à la fois une ambiguïté et une force poétiques à la narration, et l'expérience de l'écriture, toutes ancrées dans le présent. La danse, la musique et l'érotisme contribuent à mettre en relief le plaisir du corps vivant, dont la chair est fondamentale à l'expérience humaine. L'écriture aux accents féministes de LeBlanc refuse la dualité corps/esprit, et plus largement toute opposition facile tant au plan formel qu'existentiel. On lit chez la poète la «double appartenance [du corps] à l'ordre de l'objet et du sujet » (Duff et Labrosse 7), de la même façon que l'histoire acadienne ne peut être évoquée comme simple objet de mémoire : elle engage la subjectivité dans son entièreté.

LeBlanc n'accorde somme toute que peu d'attention à la question de l'identité, de la définition, ce que signalait déjà la brume inaugurale d'Alma : «c'est point la brume qui sait celle qu'elle est / pis ça l'inquiète point» (9). Il y a une nécessité de vivre qui transcende la seule recherche de récits fondateurs et qui brouille l'univocité qu'on pourrait souhaiter, comme en ont témoigné les relations interculturelles mises en scène : chez LeBlanc, conformément à l'éthique au féminin imaginée par Sing, la mémoire acadienne se révèle plurielle, d'une richesse féconde, tel un territoire à habiter au présent avec toute la sensualité dont un corps relié au monde et à l'autre est capable. Ce qui importe, c'est de créer une mémoire qui permette une joie d'exister maintenant : «Cécile hochait la tête, encore perdue dans son Rêve / c'était-il un rêve ? / le Bonheur, le Rire, la Simplicité ? » (Grand feu, 66). Engagée dans une recherche de vérité - à la fois collective et subjective - qui serait délestée de sa charge de négativité, l'écriture de LeBlanc évite à tout prix d'emprunter à ce que François Ouellet a appelé une «rhétorique victimaire » (52) en étudiant les premiers essais de Paré, qui ont fortement marqué la façon de lire les petites littératures du Canada, entre autres. Il est difficile d'expliquer pourquoi Paré évoque un «étonnant succès » au sujet d'Alma, opposant la démarche de LeBlanc aux 
«approches individuelles » de ses contemporains («Poésie acadienne contemporaine », 38). Néanmoins, il est clair que LeBlanc refuse de se laisser cantonner à la position de minoritaire, ce que son utilisation du français acadien de la Baie Sainte-Marie aurait pu laisser croire : son travail, qui allie narration à résonance volontiers mythique et éloge poétique du corps, se présente comme une manière littéraire d'appartenir de la façon la plus libre et enthousiaste au monde, et d'y jouir du temps présent. D'où l'importance de «reprendre [les] corps », scandait Prudent (105), tel que cité plus tôt, de « reprendre le désir », avait aussi affirmé son fils (55; en italiques dans le texte) : cette revendication de l'autonomie la plus intime, inscrite dans le corps et le désir, fondamentale à l'expérience humaine, constitue une façon d'affirmer sa présence la plus directe - et majoritaire - au monde, à travers la sensation partagée de vivre.

\section{Bibliographie}

Ahmed, Sara et Jackie Stacey. «Dermographies. Introduction ». Thinking Through the Skin. Dir. Sara Ahmed et Jackie Stacey. Londres et New York : Routledge, 2001. 1-17.

Duff, Christine K. et Claudia Labrosse. «Introduction». Corps écrit, corps écrivant. Le corps féminin dans les littératures francophones des Amériques. Dir. Christine K. Duff et Claudia Labrosse. Berne : Lang (Littératures de langue française), 2015. 7-17.

Labine, Marcel. «L’inachevable». Le long роѐme. Dir. Nicole Brossard. Québec : Nota bene (Cahiers du Centre Hector-De Saint-Denys-Garneau), 2011. 125-135.

Lacombe, Gilles. « Comment faire danser une histoire ». Liaison 137 (2007) : 62.

Landry, Nicolas et Nicole Lang. Histoire de l'Acadie. Deuxième édition. Québec : Septentrion, 2014.

LeBlanc, Georgette. Alma. Moncton : Perce-Neige, [2006] 2013.

---. « Alma : une performance acadienne ». Thèse de doctorat, U of Louisiana at Lafayette, 2007.

---. Amédé. Moncton : Perce-Neige, 2010.

---. «La chair des mots ». Entretien avec Marie-Louise Arsenault. Plus on est de fous, plus on lit, Ici Radio-Canada Première, 11 janvier 2013 [en ligne], consulté le 5 mai 2016.

---. «Department of French Guest Speaker : Georgette LeBlanc». Présentation suivie d'un entretien avec Joëlle Papillon et Christina Maksymetz, séquence vidéo, YouTube, McMaster Humanities, 4 novembre 2015 [en ligne], consulté le 11 mai 2016.

---. « Georgette LeBlanc... et Georgette Leblanc ». Nuit blanche 122 (2011) : 14-16. 
---. Le grand feu. Moncton : Perce-Neige, 2016.

---. « Il suffisait de le dire ». Les écrits 143 (2015) : 82.

---. « Parée ». Cousins de personne, sans date de publication [en ligne], consulté le 6 mai 2016.

---. Prudent. Moncton : Perce-Neige, 2013.

Leclerc, Catherine. " "Écriture sauvage", tradition et renouvellement en poésie acadienne ». Québec Studies 43 (2007) : 43-66.

Mousseau, Sylvie. «Georgette LeBlanc lance son $4^{\mathrm{e}}$ roman poétique ». Acadie Nouvelle, 25 avril 2016 [en ligne], consulté le 5 mai 2016.

---. «Prudent : la déportation poétique selon Georgette LeBlanc ». Acadie Nouvelle, 22 juillet 2013 [en ligne], consulté le 14 octobre 2016.

Nyela, Désiré. «LeBlanc Georgette, Amédé ». Port Acadie : revue interdisciplinaire en études acadiennes / Port Acadie : An Interdisciplinary Review in Acadian Studies 22-23 (20122013) : 263-265.

Ouellet, François. «L'héroïsme de la marge. Les essais de François Paré ». Tangence 56 (1997) : 40-65.

Papillon, Joëlle. «Plonger: Risque, désir, mobilité et création au féminin chez Georgette LeBlanc ». Voix plurielles XIII.2 (2016) : dans ce même numéro.

Paré, François. La distance habitée. Ottawa : Le Nordir, 2003.

---. «La poésie acadienne contemporaine ». Nuit blanche, magazine littéraire 115 (2009) : 3438.

Ricœur, Paul. «La fonction narrative ». La narrativité. Dir. Dorian Tiffeneau. Paris : CNRS (Phénoménologie et herméneutique), 1980. 49-68.

---. La mémoire, l'histoire, l'oubli. Paris : Seuil, 2000.

Saada, Lamia. «Le dedans et le dehors dans Alma - Une lecture d'Alma de Georgette LeBlanc ». Port Acadie: revue interdisciplinaire en études acadiennes / Port Acadie : An Interdisciplinary Review in Acadian Studies 18-19 (2010-2011) : 51-61.

Sing, Pamela V. «Mémoire, sexualité et déconstruction ». La parole mémorielle des femmes. Dir. Lucie Hotte et Linda Cardinal. Montréal: Remue-ménage, 2002. 157-170. 


\section{NOTES}

${ }^{1}$ Commencement.

${ }^{2}$ Je m'inspire ici de la partition qu'utilise Ricœur dans le chapitre "Mémoire personnelle, mémoire collective » (112-163) de La mémoire, l'histoire, l'oubli, conformément à son cadre de réflexion phénoménologique.

${ }^{3}$ C'est d'ailleurs ce qu'elle admire chez son contemporain Serge Patrice Thibodeau, poète acadien : «Serge Patrice, / Les fragments. La relation causale. T'as compris. T'as senti. T’es sorti. De l'enfer, du chemin linéaire et déterminé. De l'histoire répétée. Sorti du carcan de ce qu'on a été dit qui devrait marcher. Tes fragments ouvrent une porte brisée qu'était restée fermée. Qu'empêchait de marcher » («Suffisait de le dire », 82).

${ }^{4}$ Pour en savoir plus sur les motivations de LeBlanc à créer ce personnage féminin inédit et le projet littéraire et sociologique derrière Alma, je recommande la lecture de sa thèse de doctorat en études francophones, intitulée «Alma : une performance acadienne », University of Louisiana at Lafayette.

${ }^{5}$ Nénuphar.

${ }^{6}$ Bouger.

${ }^{7}$ La thèse de doctorat de LeBlanc fait état des projets de théâtre corporel et de danse ayant précédé la création du personnage d'Alma et elle mentionne que ces performances étaient une façon de "raconter [s]on intimité à l'ensemble de [s]a communauté affective [acadienne, francophone et anglophone] sans avoir besoin de négocier la parole $»(14)$.

${ }^{8}$ En 1755, deux cent trente-deux Acadiens sont embarqués à bord du Pembroke, accosté à Port-Royal, pour être déportés sur la côte Est américaine, mais ils se révoltent et prennent le contrôle du bateau. Ils reviendront plus tard au port de Saint-Jean (Nouveau-Brunswick).

${ }^{9}$ Cette fin lyrique a été ajoutée après le dépôt du manuscrit à l'éditeur, en partie parce que le poète acadien JeanPhilippe Raîche, ayant participé à l'édition de Prudent, a déploré que la première version «manquait de Georgette LeBlanc » («Department of French »).

${ }^{10}$ «Si j'avais le choix moi / ej serais un pissenlit avant d'être une rose / ej pourrais courir partout » (Alma, 87). 